\title{
Desenvolvimento sustentável municipal e financiamento bancário: análise do Programa Municípios Verdes Paraenses
}

\section{Municipal sustainable development and bank financing: analysis of the Green Municipalities Program in Pará}

\author{
Vanessa Paiva Costa Vale ${ }^{a}$ \\ Marco Valério de Albuquerque Vinagre ${ }^{b}$ \\ Marco Aurélio Arbage Lobo ${ }^{c}$ \\ Leonardo Augusto Lobato Bello ${ }^{d}$ \\ Alberto Carlos de Melo Lima
}

\begin{abstract}
a Mestre em Desenvolvimento e Meio Ambiente Urbano pela Universidade da Amazônia (Unama) e Assessora de Arquitetura e Engenharia do Centro de Serviços Coorporativos e Patrimônio do Banco do Brasil, Belém, PA, Brasil. End. Eletrônico: nessa@bb.com.br
\end{abstract}

${ }^{b}$ Doutor em Engenharia de Recursos Naturais da Amazônia pela Universidade Federal do Pará (UFPA) e Professor Efetivo do Departamento de Arquitetura e de Engenharia da Universidade da Amazônia (Unama), Belém, PA, Brasil.

End. Eletrônico: valeriovinagre@gmail.com

'Doutor em Desenvolvimento Socioambiental, Universidade Federal do Pará (UFPA) e Professor Efetivo do Departamento de Arquitetura e de Engenharia da Universidade da Amazônia (Unama), Belém, PA, Brasil. End. Eletrônico: lobo2502@gmail.com

${ }^{d}$ Doutor em Engenharia Civil - Geotecnia pela Pontifícia Universidade Católica do Rio de Janeiro (PUC-RIO) e Professor Titular e coordenador do Programa de Mestrado em Desenvolvimento e Meio Ambiente Urbano da Universidade da Amazônia (Unama), Belém, PA, Brasil. End. Eletrônico: leonardo.bello@unama.br

${ }^{e}$ Doutor em Engenharia Civil com ênfase em Hidráulica e Saneamento pela Escola de Engenharia de São Carlos Universidade de São Paulo (EESC/USP) e Professor Efetivo do Programa de Pós-Graduação da Universidade do Estado do Pará (UEPA), Belém, Pará, Brasil. End. Eletrônico: acmlima@gmail.com

doi:10.18472/SustDeb.v9n2.2018.26151

Recebido em 16.06.2017

Aceito em 23.01.2018

ARTIGO - VARIA

\section{RESUMO}

A preocupação com o desenvolvimento sustentável no mundo e no Brasil possibilitou a criação do Programa Municípios Verdes no Pará que combate o desmatamento no estado e fomenta a gestão ambiental. Este trabalho objetiva analisar a relação do nível de sustentabilidade dos municípios verdes paraenses com os financiamentos bancários para a promoção do desenvolvimento sustentável. A pesquisa foi realizada por meio da correlação de Pearson, a partir da criação dos Índices de Desenvolvimento Sustentável de 13 municípios com os financiamentos bancários liberados em 2010. Os resultados obtidos apresentaram índices entre 0,3773, considerado como nível de Alerta, e 0,5310 como nível Aceitável, o que evidencia a fraca relação de maior nível de sustentabilidade municipal com a obtenção de financiamentos bancários direcionados a esse fim. $O$ estudo aponta a necessidade dos bancos reverem suas linhas de financiamentos para o desenvolvimento sustentável municipal para a promoção do desenvolvimento econômico, social, cultural e ambiental.

Palavras-chave: Desenvolvimento Sustentável; Indicadores de Sustentabilidade; Municípios Verdes; Financiamentos Bancários. 


\begin{abstract}
The concern over sustainable development in the world and in Brazil has made possible the creation of The Green Municipality Program in the State of Pará. The program combats deforestation in the state and encourages environmental resource management. The objective of this study is to analyze the level of sustainability of the green municipalities of Pará in relation to bank financing to promote sustainable development. The study was conducted applying the Pearson Correlation after creating sustainable development indexes for 13 municipalities that received bank financing in 2010. The results obtained demonstrated indexes between 0.3773 , considered as a level of alert, and 0.5310 , as an acceptable level. The results show a weak correlation of higher level of municipal sustainability with the access to bank financing for this purpose. The study points to the need for banks to revise their current guidelines for financing municipal sustainable development to promote economic, social, cultural, and environmental development.
\end{abstract}

Keywords: Sustainable Development; Sustainability Indicators; Green Municipalities; Banks Financings.

\title{
1 INTRODUÇÃO
}

O termo "desenvolvimento sustentável" vem sendo sistematicamente usado nas convenções internacionais sobre meio ambiente e desenvolvimento. Repete-se exaustivamente nos documentos oficiais e é inserido na formulação de políticas públicas mundiais. Apesar de todos os esforços das políticas governamentais, a proposta do ambientalismo moderado ${ }^{1}$ não tem conseguido reduzir desequilíbrios socioespaciais, resultantes das políticas de concentração econômica, excludentes e espacialmente segregadoras, especialmente em países em desenvolvimento.

Nos países desenvolvidos, observa-se que as propostas para o desenvolvimento sustentável vêm se tornando mais efetivas, por meio de grandes aportes de recursos, em decorrência do estágio de desenvolvimento que se encontram, o que permite maior convergência social nas suas políticas territoriais (BANCO MUNDIAL, 2008).

No Brasil, principalmente na Amazônia Legal, que compreende os estados do Acre, Amapá, Amazonas, Mato Grosso, Pará, Rondônia, Roraima, Tocantins ( $98 \%$ da área do estado), Maranhão (79\%) e Goiás $(0,8 \%)$, a preocupação recai sobre o processo de desmatamento, já que boa parte dos estudos sobre desmatamento de florestas tropicais centraliza-se nessa região, pois representa grande ameaça para o acervo de biodiversidade e, principalmente, para a prestação de serviços de manutenção ambiental.

Segundo Oliveira (2011), em 1980, o desmatamento na região alcançava cerca de $300 \mathrm{mil} \mathrm{km²}$, o equivalente a $6 \%$ da área total, enquanto que, em 2007, houve um aumento significante no desmatamento, cerca de $732 \mathrm{mil} \mathrm{km}^{2}$ foram desmatados, o que corresponde a quase $15 \%$ da área total da região.

Para Brasil (2007), o Plano Amazônia Sustentável (PAS) serve de diretriz para o desenvolvimento sustentável da Amazônia brasileira, com objetivo de promover o ordenamento territorial e a gestão ambiental, de modo a possibilitar o combate à grilagem, a resolução de conflitos fundiários e destinação de terras públicas, o controle sobre a exploração ilegal e predatória de recursos naturais e a proteção dos ecossistemas regionais.

O estado do Pará conta com uma área de 1,25 milhão de $\mathrm{Km} 2$, cerca de 7,6 milhões de habitantes, distribuídos em 144 municípios, grandes estoques de recursos naturais, uma vasta rede hidrográfica ( $1 / 5$ do potencial hidrelétrico nacional) e ricas e diversas jazidas minerais. Essas riquezas potenciais, no entanto, não têm gerado os benefícios socioeconômicos e melhoria de qualidade de vida da população, de forma que, em 2009, o Pará detinha um PIB per capita inferior a US\$ 5 mil (metade da média nacional).

De modo geral, isso ocorre porque a economia paraense baseia-se em grandes projetos de mineração e energia, geradores de alto impacto socioambiental e baixo retorno tributário, e em atividades rurais 
com baixo valor agregado e alta emissão de carbono. Tais atividades, em especial a pecuária, foram desenvolvidas à custa de um forte processo de desmatamento, resultando na alteração de $21 \%$ do território do estado, fazendo do Pará, ao lado do Mato Grosso, um dos líderes do desmatamento na Região Amazônica (PMV, 2016).

Para combater o desmatamento no estado do Pará, foi criado o Programa Municípios Verdes (PMV), uma política de desenvolvimento municipal, alinhado com as políticas públicas federais e estaduais de combate ao desmatamento e promoção do desenvolvimento sustentável da Amazônia. Especialmente preconizada pelo PAS, visa combater o desmatamento no estado e fomentar a gestão ambiental, com foco em pactos locais, na busca de um compromisso maior com os impactos socioambientais que cada empreendimento gera na região onde está inserido (PMV, 2016).

A discussão acerca dessa temática é importante para estabelecer a diferença entre as abordagens do crescimento econômico associado à degradação ambiental, promovendo novas formas de operacionalizar esse conceito, tais como a criação de indicadores que possam contribuir para um planejamento do desenvolvimento local de forma mais sustentável (VALE, 2017).

\section{SUSTENTABILIDADE E O DESENVOLVIMENTO SUSTENTÁVEL}

A possibilidade de construir o conceito de desenvolvimento sustentável deve levar em consideração os princípios extraídos dos recentes avanços das teorias científicas sobre a sustentabilidade, uma vez que a insustentabilidade é resultante do conhecimento anterior, inadequado, de convivência com o meio ambiente.

Para compor a base para a construção da sustentabilidade, Rohde (1999) relacionou princípios filosóficos: Princípio de Contingência, que reflete o novo não necessário; Princípio de Complexidade, que é a oposição ao reducionismo praticado; Princípio de Sistêmica, que possui uma abordagem holística quanto à totalidade; Princípio de Recursividade, baseado na reorganização permanente; Princípio de Conjunção, que permeia todos os paradigmas científicos novos; e o Princípio de Interdisciplinaridade, que é a correção para o estilhaçamento da razão nas diversas racionalidades hoje existentes.

É importante ressaltar que esses princípios são extraídos da teoria do conhecimento e dos novos paradigmas científicos e, portanto, constituem parte do aparato conceitual disciplinar para uma abordagem sustentável. Princípios éticos, sociais e econômicos deverão igualmente entrar em formação das novas propostas de desenvolvimento da sociedade (ELY, 1992).

No decorrer dos debates internacionais sobre a sustentabilidade do planeta, surgiu a Agenda 21, que foi um documento resultado da conferência ECO-92, composto por 40 capítulos com um programa de ação voltado ao desenvolvimento sustentável para o século XXI. Portanto, é um documento que estabelece o comprometimento de cada país em buscar novas soluções para os problemas sociais e ambientais. De acordo com essa Agenda, a procura por novas tecnologias tem o desafio de utilizar recursos naturais de forma mais segura e mais eficiente que as atuais. Ademais, outros pontos importantes da agenda são observados, como: a substituição dos recursos não renováveis pelos renováveis, eliminação de substâncias tóxicas, redução da poluição e produção mais limpa (BARBIERI, 2001).

Portanto, conceituar o desenvolvimento da sustentabilidade é um processo contínuo e complexo de reavaliação crítica entre a sociedade e seu meio natural. Fialho et al. (2008) afirmam que a sustentabilidade pode ser analisada por diferentes dimensões (econômica, social e ambiental) e que estas, embora apresentem similaridades, não podem ser apresentadas de forma isolada, ou seja, não se pode tratar a dimensão ambiental sem levar em consideração a econômica e a social. Van Bellen (2005) afirma que a inclusão de mais dimensões nos modelos de mensuração de resultados é uma escolha da sociedade, das organizações, das comunidades e dos indivíduos, todos engajados com o mesmo propósito. Observa-se que muitas questões e dimensões estão atreladas ao conceito de desenvolvimento sustentável. Para apontar esse desenvolvimento, foram construídos indicadores com potencial de direcionar, de forma mais perceptível, uma tendência ou fenômeno ainda não diagnosticado. 


\section{FINANCIAMENTOS BANCÁRIOS E O DESENVOLVIMENTO LOCAL}

As instituições financeiras vêm apresentando um papel importante na liberação de financiamentos para o desenvolvimento sustentável. Em 1992, na cidade de New York (US), foi assinada a Declaração dos Bancos para o Meio Ambiente e Desenvolvimento com a participação de aproximadamente 30 bancos comerciais, com sede em 23 países, assumindo o compromisso de vincular suas operações financeiras aos princípios comuns de proteção ao meio ambiente (SANTOS, 1999).

Para Braga (2014), a Declaração dos Bancos para o Meio Ambiente e Desenvolvimento teve como objetivo a formalização das instituições financeiras na adoção de ações preventivas que evitem os riscos envolvidos em falhas de seus clientes quanto à questão ambiental. Nessas ações estão incluídos projetos de implantação de práticas e avaliações que contribuem para atingir o desenvolvimento sustentável.

Em 1995, foi firmado o Protocolo Verde, um acordo entre o governo brasileiro e os principais bancos públicos federais, considerado a versão brasileira da Declaração dos Bancos para o Meio Ambiente e Desenvolvimento. Por meio dele, os bancos signatários se comprometeram a incorporar a variável ambiental na concessão de crédito e de benefícios fiscais.

De acordo com a base de dados do Laboratório de Estudos em Moeda e Território (LEMTe), extraída do site do Banco Central, são demonstrados no Quadro 1 os tipos de financiamentos disponibilizados para os municípios no Brasil.

Quadro 1 - Tipos de financiamentos da base de dados do Laboratório de Estudos em Moeda e Território (LEMTe)

\begin{tabular}{|c|c|c|}
\hline № da conta & Nome da conta & Descrição \\
\hline 161 & $\begin{array}{c}\text { EMPRÉSTIMOS E TÍTULOS DESCON- } \\
\text { TADOS }\end{array}$ & $\begin{array}{l}\text { Interpretada como representando empréstimos de } \\
\text { curto, sobretudo financiando o consumo. }\end{array}$ \\
\hline 162 & FINANCIAMENTOS & $\begin{array}{l}\text { Representa o total de financiamento (conta 162) } \\
\text { menos as contas de financiamento especificadas } \\
\text { acima. }\end{array}$ \\
\hline $163+165$ & FINANCIAMENTOS AGRICULTURA & $\begin{array}{l}\text { Soma das contas voltadas ao financiamento da } \\
\text { agricultura. }\end{array}$ \\
\hline $164+166$ & FINANCIAMENTOS PECUÁRIOS & $\begin{array}{l}\text { Soma das contas voltadas ao financiamento da } \\
\text { pecuária. }\end{array}$ \\
\hline 167 & $\begin{array}{l}\text { FINANCIAMENTOS AGROINDUS- } \\
\text { TRIAIS }\end{array}$ & $\begin{array}{c}\text { Registra as operações realizadas sob a modalidade } \\
\text { de financiamento agroindustrial, a pessoas físicas e } \\
\text { jurídicas. }\end{array}$ \\
\hline 169 & FINANCIAMENTOS IMOBILIÁRIOS & $\begin{array}{c}\text { Registra as operações realizadas sob a modalidade } \\
\text { de financiamento imobiliário. }\end{array}$ \\
\hline
\end{tabular}

Fonte: Elaborado pela autora a partir do Banco Central (2016).

\section{PROGRAMA MUNICÍPIOS VERDES - PMV}

O PMV foi transformado em projeto de governo do estado do Pará em março de 2011, por meio do Dec. Estadual no 54/2011, após a iniciativa do Fundo Vale (instrumento de ação da empresa Vale nos territórios onde ela atua) em 2008, como ferramenta para auxiliar os municípios que compunham a lista dos maiores desmatadores da Amazônia, divulgada pelo site do Ministério do Meio Ambiente. Tem como objetivo combater o desmatamento no estado e fortalecer a produção rural sustentável por meio de ações estratégicas de ordenamento ambiental e fundiário. E também tem o compromisso de fomentar a gestão ambiental, com foco em pactos locais, no monitoramento do desmatamento, na 
implantação do Cadastro Ambiental Rural (CAR) e na estruturação da administração voltada ao meio ambiente dos municípios participantes.

Os financiamentos do PMV são oriundos do Fundo Amazônia (FA), criado pelo Governo Federal em 2008 e gerenciado pelo Banco Nacional de Desenvolvimento Econômico e Social (BNDES). O FA tem como objetivo captar doações para investimentos não reembolsáveis em ações de prevenção, monitoramento, combate ao desmatamento, promoção da conservação e do uso sustentável das florestas no Bioma Amazônia.

Para De Freitas (2013), há muitas críticas em relação ao Fundo Amazônia e aos recursos oriundos do BNDES, pois nota-se uma excessiva burocratização, seja no processo ou na liberação de recursos. Um dos fatores é a demora na obtenção da aprovação de projetos, de cerca de um a dois anos, ou a não aprovação. Já para Gonçalves (2016), a burocracia aparente para a liberação de recursos financeiros por parte do BNDES está na sua condição de instituição financeira, responsabilizando-se pelo Fundo que lhe foi confiado.

Os municípios do Pará aderem ao Programa Municípios Verdes (PMV) por meio de assinatura de termo com o Ministério Público Federal (MPF), no qual se comprometem com metas monitoradas pela coordenação do PMV: elaboração do pacto local contra o desmatamento que envolva a sociedade e o governo municipal; criação de grupo de trabalho municipal visando combater o desmatamento; monitoramento em campo e a notificação ao PMV dos focos de desmatamento ilegal; manutenção do desmatamento anual abaixo de $40 \mathrm{~km}^{2}$; cadastro de $80 \%$ da área municipal do Cadastro Ambiental Rural (CAR); não aparecer na lista do MMA de maiores desmatadores da Amazônia e a introdução de noções de educação ambiental na rede municipal de ensino. O cumprimento das metas habilita os municípios a receberem benefícios como o desembargo ambiental, incentivos fiscais e prioridade na aplicação de recursos públicos (WHATELY; CAMPANILI, 2013).

Há 144 municípios no estado do Pará, sendo que dos 107 municípios que aderiram ao PMV, apenas 15 municípios já conseguiram a Certificação de Municípios Verdes, conforme demonstrado na Tabela 1.

Tabela 1 - Lista dos Municípios Verdes Paraenses até agosto/2016.

\begin{tabular}{|c|c|c|}
\hline № & Municípios Verdes & Data da Certificação \\
\hline 1 & Paragominas & $05 / 11 / 2009$ \\
\hline 2 & Tailândia & $06 / 01 / 2010$ \\
\hline 3 & Santa Maria das Barreiras & $25 / 01 / 2010$ \\
\hline 4 & Tucumã & $13 / 07 / 2010$ \\
\hline 5 & Xinguara & $13 / 07 / 2010$ \\
\hline 6 & Óbidos & $13 / 08 / 2010$ \\
\hline 7 & Redenção & $29 / 11 / 2010$ \\
\hline 8 & Canãa dos Carajás & $20 / 10 / 2011$ \\
\hline 9 & Juruti & $15 / 03 / 2012$ \\
\hline 10 & Dom Eliseu & $05 / 07 / 2012$ \\
\hline 11 & Santana do Araguaia & $21 / 08 / 2012$ \\
\hline 12 & Santarém & $18 / 03 / 2013$ \\
\hline 13 & Brasil Novo & $15 / 10 / 2013$ \\
\hline 14 & Cumaru do Norte & $28 / 01 / 2014$ \\
\hline 15 & Ulianópolis & $03 / 07 / 2014$ \\
\hline
\end{tabular}

Fonte: Adaptado do PMV (2016). 


\section{5 ÁREA PESQUISADA E PROCEDIMENTOS METODOLÓGICOS}

A escolha dos municípios ocorreu a partir da relação dos municípios paraenses que já conseguiram a certificação de municípios verdes, publicada no site do $\mathrm{PMV}^{2}$. Do total de 15 municípios paraenses que receberam a certificação verde, foram selecionados 11 que possuíam de três a oito instituições bancárias no período de 2010 e 2015.

Foram escolhidos, também, dois municípios que não possuem a certificação verde pelo PMV: Altamira e Tucuruí, ambos municípios importantes para análise do desenvolvimento sustentável por serem polos geradores de energia protegidos ambientalmente. Com essa característica, esses municípios servem como base de equiparação em relação aos municípios verdes pesquisados. Assim, o presente estudo vai calcular o Índice de Desenvolvimento Sustentável para Municípios (IDSM) de 13 municípios paraenses que possuem de 3 a 8 instituições financeiras, relacionados na Tabela 2:

Tabela 2 - Municípios Paraenses e as quantidades de Instituições Financeiras.

\begin{tabular}{|c|c|c|}
\hline Municípios Paraenses & $\begin{array}{c}\text { Possui } \\
\text { Certificação Verde }\end{array}$ & $\begin{array}{c}\text { No de instituiçães financeiras } \\
\text { nos micípios no período entre } \\
\text { 2010 e 2015. }\end{array}$ \\
\hline Altamira & Não & 8 \\
\hline Canãa dos Carajás & Sim & 5 \\
\hline Dom Eliseu & Sim & 4 \\
\hline Juruti & Sim & 4 \\
\hline Óbidos & Sim & 7 \\
\hline Paragominas & Sim & 7 \\
\hline Redenção & Sim & 3 \\
\hline Santana do Araguaia & Sim & 8 \\
\hline Santarém & Sim & 5 \\
\hline Tailândia & Sim & 3 \\
\hline Tucumã & Sim & 7 \\
\hline Tucuruí & Não & 5 \\
\hline Xinguara & Sim & \\
\hline
\end{tabular}

Fonte: Autora (2016)

Participaram da pesquisa oito instituições financeiras (quatro públicas e quatro privadas), atuantes no Departamento de Operações do Banco Central do Brasil e presentes nos municípios avaliados (Tabela 3). Seus nomes foram preservados, sem perda da relevância das informações, que são públicas e estão disponíveis nos websites e relatórios de sustentabilidade dos bancos na internet, podendo, portanto, serem rastreadas e recuperadas por qualquer pessoa ou instituição.

Tabela 3 - Relação das Instituições Financeiras presentes nos municípios.

\begin{tabular}{|c|c|c|c|c|c|c|c|c|}
\hline \multirow{2}{*}{ Cidade } & \multicolumn{7}{|c|}{ Instituições Bancárias (2010 a 2015) } \\
\cline { 2 - 11 } & PB1 & PB2 & PB3 & PB4 & PV1 & PV2 & PV3 & PV4 \\
\hline Altamira & $\mathrm{X}$ & $\mathrm{X}$ & $\mathrm{X}$ & $\mathrm{X}$ & $\mathrm{X}$ & $\mathrm{X}$ & $\mathrm{X}$ & $\mathrm{X}$ \\
\hline Canãa dos Carajás & $\mathrm{X}$ & $\mathrm{X}$ & $\mathrm{X}$ & $\mathrm{X}$ & $\mathrm{X}$ & & & \\
\hline Dom Eliseu & $\mathrm{X}$ & $\mathrm{X}$ & $\mathrm{X}$ & $\mathrm{X}$ & $\mathrm{X}$ & & & \\
\hline Juruti & & $\mathrm{X}$ & $\mathrm{X}$ & $\mathrm{X}$ & $\mathrm{X}$ & & & \\
\hline Óbidos & $\mathrm{X}$ & $\mathrm{X}$ & $\mathrm{X}$ & & $\mathrm{X}$ & & & \\
\hline Paragominas & $\mathrm{X}$ & $\mathrm{X}$ & $\mathrm{X}$ & $\mathrm{X}$ & $\mathrm{X}$ & $\mathrm{X}$ & $\mathrm{X}$ & \\
\hline Redenção & $\mathrm{X}$ & $\mathrm{X}$ & $\mathrm{X}$ & $\mathrm{X}$ & $\mathrm{X}$ & $\mathrm{X}$ & $\mathrm{X}$ & \\
\hline Santana do Araguaia & & $\mathrm{X}$ & $\mathrm{X}$ & & $\mathrm{X}$ & & & \\
\hline
\end{tabular}




\begin{tabular}{|c|c|c|c|c|c|c|c|c|}
\hline \multirow{2}{*}{ Cidade } & \multicolumn{7}{|c|}{ Instituições Bancárias (2010 a 2015) } \\
\cline { 2 - 11 } & PB1 & PB2 & PB3 & PB4 & PV1 & PV2 & PV3 & PV4 \\
\hline Santarém & $\mathrm{X}$ & $\mathrm{X}$ & $\mathrm{X}$ & $\mathrm{X}$ & $\mathrm{X}$ & $\mathrm{X}$ & $\mathrm{X}$ & $\mathrm{X}$ \\
\hline Tailândia & $\mathrm{X}$ & $\mathrm{X}$ & $\mathrm{X}$ & $\mathrm{X}$ & $\mathrm{X}$ & & & \\
\hline Tucumã & & $\mathrm{X}$ & $\mathrm{X}$ & & $\mathrm{X}$ & & & \\
\hline Tucuruí & $\mathrm{X}$ & $\mathrm{X}$ & $\mathrm{X}$ & $\mathrm{X}$ & $\mathrm{X}$ & $\mathrm{X}$ & $\mathrm{X}$ & \\
\hline Xinguara & $\mathrm{X}$ & $\mathrm{X}$ & $\mathrm{X}$ & $\mathrm{X}$ & $\mathrm{X}$ & & & \\
\hline
\end{tabular}

Fonte: Elaborada pela autora a partir do Banco Central (2016).

Nota: $P B=$ pública e $P V=$ privada.

Para calcular o IDSM, Martins e Cândido (2008) basearam-se nas referências propostas de Waquil et al. (2006) para um conjunto de seis dimensões e variáveis definidas, que se desdobram em 44 indicadores de forma multidimensional, de acordo com o Quadro 2.

Quadro 2 - Dimensões e variáveis do IDSM.

\begin{tabular}{|c|c|}
\hline Dimensão & Variáveis \\
\hline \multirow{13}{*}{ SOCIAL } & Esperança de vida ao nascer \\
\hline & Mortalidade infantil \\
\hline & Prevalência da desnutrição total \\
\hline & Imunização contra doenças infecciosas infantis \\
\hline & Oferta de serviços básicos de saúde \\
\hline & Escolarização \\
\hline & Alfabetização \\
\hline & Escolaridade \\
\hline & Analfabetismo funcional \\
\hline & Famílias atendidas com programas sociais \\
\hline & Adequação de moradia nos domicílios \\
\hline & Mortalidade por homicídio \\
\hline & Mortalidade por acidente de transporte \\
\hline \multirow{5}{*}{ DEMOGRÁFICA } & Crescimento da população \\
\hline & Razão entre a população urbana e rural \\
\hline & Densidade demográfica \\
\hline & Razão entre a população masculina e feminina \\
\hline & Distribuição da população por faixa etária \\
\hline \multirow{7}{*}{ ECONÔMICA } & Produto Interno Bruto per capita \\
\hline & Participação da indústria no PIB \\
\hline & Saldo da balança comercial \\
\hline & Renda familiar per capita em salários mínimos \\
\hline & Renda per capita \\
\hline & Rendimentos provenientes do trabalho \\
\hline & Índice de Gini de distribuição do rendimento \\
\hline \multirow{6}{*}{ POLÍTICO-INSTITUCIONAL } & Despesas por função \\
\hline & Acesso a serviço de telefonia fixa \\
\hline & Participação nas eleições \\
\hline & Número de conselhos municipais \\
\hline & Número de acessos à justiça \\
\hline & Transferências intergovernamentais da União \\
\hline
\end{tabular}




\begin{tabular}{|l|l|}
\hline \multicolumn{1}{|c|}{ Dimensão } & \multicolumn{1}{|c|}{ Variáveis } \\
\hline \multirow{4}{*}{ AMBIENTAL } & Qualidade das águas \\
\cline { 2 - 3 } & Tratamento das águas: tratada em ETAs e por desinfecção \\
\cline { 2 - 3 } & Consumo médio per capita de água \\
\cline { 2 - 3 } & Acesso ao sistema de abastecimento de água \\
\hline & Tipo de esgotamento sanitário por domicílio \\
\hline & Acesso à coleta de lixo urbano e rural \\
\hline \multirow{4}{*}{ CULTURAL } & Quantidade de bibliotecas \\
\cline { 2 - 3 } & Quantidade de museus \\
\hline & Quantidade de ginásios de esportes e estádios \\
\hline & Quantidade de cinemas \\
\hline & Quantidade de unidades de ensino superior \\
\cline { 2 - 3 } & Quantidade de teatros ou salas de espetáculos \\
\hline & Quantidade de centros culturais \\
\hline
\end{tabular}

Fonte: Adaptado de Martins e Cândido (2008).

Martins e Cândido (2008) ajustam os valores das variáveis para uma escala de variação entre 0 (zero) e 1 (um), sendo 0 (zero) o valor mínimo e 1 (um) o valor máximo. Em seguida, classificou-se o tipo de relação das variáveis como Positivas e Negativas, permitindo uma adequação mais justa desses índices, ao identificar o comportamento da variável que favorece ou desfavorece o desenvolvimento sustentável. Na relação Positiva, quanto maior o indicador, melhor será o índice e, quanto menor o indicador, pior será o índice. Enquanto que na relação Negativa, quanto maior o indicador, pior o índice e, quanto menor o indicador, melhor será o índice. Para Martins e Cândido (2008), para a operacionalização do cálculo do índice são utilizadas as seguintes equações:

Equação quando a relação for Positiva.

$$
\mathbf{I}=\frac{(\boldsymbol{x}-\boldsymbol{m})}{(M-m)}
$$

Equação quando a relação for Negativa.

$$
\begin{gathered}
\text { I }=\frac{(\boldsymbol{M}-\boldsymbol{x})}{(\boldsymbol{M}-\boldsymbol{m})} \\
\text { Onde: } \\
\text { I = índice calculado para cada município } \\
x=\text { valor de cada variável em cada estado e município } \\
m=\text { valor mínimo identificado nessas localidades } \\
M=\text { valor mínimo identificado nessas localidades }
\end{gathered}
$$

Após calcular os índices referentes a cada variável, os dados passam a ser a

gregados por dimensão, por meio da média aritmética, chegando-se aos resultados do IDSM por dimensão: social, demográfica, econômica, político-institucional, ambiental e cultural dos municípios. Para obtenção do IDSM final, calcula-se a média aritmética das dimensões e, para a classificação dos índices referente a cada variável, é utilizado um conjunto de cores que correspondem a uma escala definida, caracterizando o nível de sustentabilidade local, conforme demonstrado na Tabela 4. 
Tabela 4 - Classificação e representação do IDSM.

\begin{tabular}{|c|c|c|}
\hline Índice (0-1) & Coloração & $\begin{array}{c}\text { Nível de } \\
\text { Sustentabilidade }\end{array}$ \\
\hline $0,0000-0,2500$ & & CRÍTICO \\
\hline $0,2501-0,5000$ & & ALERTA \\
\hline $0,5001-0,7500$ & & ACEITÁVEL \\
\hline $0,7501-1,0000$ & & IDEAL \\
\hline
\end{tabular}

Fonte: Adaptado de Martins e Cândido (2008).

A classificação estabelecida por Martins e Cândido (2008) apresenta os índices de sustentabilidade com as respectivas cores e a classificação que se utiliza em uma escala, que varia de 0 (zero) a 1 (um). Assim, a cor vermelha com valores entre 0,0000 e 0,2500 caracteriza um nível crítico de sustentabilidade; a cor laranja, com valores entre 0,2501 e 0,5000, caracteriza um nível de sustentabilidade em alerta; a cor verde-claro, com valores entre 0,5001 e 0,7500, caracteriza um nível de sustentabilidade aceitável; e a cor verde brilhante, com valores entre 0,7501 e 1,0000, caracteriza um nível de sustentabilidade ideal. Essa representação é realizada para todas as 44 variáveis, nas seis dimensões e para o IDSM final.

Para quantificar o volume de recursos liberados de financiamentos no ano de 2010, buscou-se uma combinação de dados financeiros obtidos da base de Estatística Bancária por município (Estban), retirada do site do Banco Central, por meio do programa Sisbacen, disponibilizado na internet. Essa base de dados se refere ao balancete agregado dos bancos por municípios, onde há, pelos menos, uma agência bancária.

Para expressar os recursos liberados no ano de 2010 por município, foi calculada a variável do recurso monetário por meio da somatória dos financiamentos liberados mensalmente, ao longo do ano, pelo total da população dos municípios da pesquisa. Essa análise de financiamentos liberados pelas instituições financeiras públicas e privadas evidencia algumas características quanto ao desenvolvimento local.

Para investigar a relação da sustentabilidade municipal retratada pelo IDSM com os volumes dos financiamentos bancários para a promoção do desenvolvimento sustentável dos municípios paraenses, foi utilizado o cálculo de correlação de Pearson. Essa metodologia apresenta um modelo de correlação de variáveis que permite avaliar a influência negativa ou positiva entre duas variáveis de grandezas distintas ou não.

Para Pearson (1920), o modelo de correlação de variáveis, parte da seguinte premissa: se duas variáveis podem se movimentar conjuntamente, uma correlação pode ser estabelecida. Se a variável " $x$ " tende a aumentar quando " $y$ " cresce, existe uma correlação positiva, isso indica que a elevação de uma variável afeta a outra positivamente. Se a variável " $x$ " tende a cair quando " $y$ " aumenta, existe uma correlação negativa, ou seja, uma correlação negativa indica movimentos opostos.

Para Cohen (1988), o coeficiente "r" varia entre 0 e 1, portanto, a correlação pode ser estabelecida a partir das seguintes situações analíticas:

Tabela 5 - Intervalo do coeficiente de correlação de Pearson.

\begin{tabular}{|c|c|}
\hline Correlação & Intervalo $(r)$ \\
\hline Ausência & 0,000 a 0,099 \\
\hline Fraca & 0,100 a 0,299 \\
\hline Média & 0,300 a 0,499 \\
\hline Forte & 0,500 a 1,000 \\
\hline
\end{tabular}

Fonte: Adaptada de Cohen (1988). 


\section{ANÁLISE E INTERPRETAÇÃO DOS RESULTADOS}

Das seis dimensões analisadas, serão apresentadas apenas três dimensões (social, econômica e ambiental) para demonstrar a interpretação dos resultados. Com base nos valores encontrados, a Tabela 6 compõe a dimensão social.

Tabela 6 - Nível de Sustentabilidade SOCIAL dos municípios por variável.

\begin{tabular}{|c|c|c|c|c|c|}
\hline \multirow{2}{*}{$\begin{array}{c}\text { DIMENSÃO SOCIAL } \\
13 \text { municípios do estado do Pará } \\
\text { VARIÁVEIS }\end{array}$} & \multicolumn{5}{|c|}{$\begin{array}{c}\text { Nível de Sustentabilidade SOCIAL dos } 13 \text { municípios } \\
\text { paraenses, por variável (unidade) }\end{array}$} \\
\hline & IDEAL & ACEITÁVEL & ALERTA & CRITICA & $\begin{array}{c}\text { SEM } \\
\text { INFORM. }\end{array}$ \\
\hline Esperança de vida ao nascer & 4 & 5 & 4 & 0 & 0 \\
\hline Mortalidade infantil & 10 & 3 & 0 & 0 & 0 \\
\hline Prevalência da desnutrição total & 10 & 2 & 0 & 1 & 1 \\
\hline \multicolumn{6}{|l|}{$\begin{array}{l}\text { Imunização (doenças infecciosas } \\
\text { infantis) }\end{array}$} \\
\hline Tetraviral & 1 & 1 & 2 & 6 & 9 \\
\hline Tríplice & 4 & 6 & 2 & 0 & 1 \\
\hline Poliomielite & 12 & 1 & 0 & 0 & 0 \\
\hline BCG & 4 & 5 & 4 & 0 & 0 \\
\hline \multicolumn{6}{|l|}{$\begin{array}{l}\text { Oferta de serviços básicos de } \\
\text { saúde }\end{array}$} \\
\hline $\begin{array}{l}N^{\circ} \text { de procedimentos básicos de } \\
\text { saúde } p / \text { hab. }\end{array}$ & 11 & 2 & 0 & 0 & 0 \\
\hline $\mathrm{N}^{\circ}$ de médicos por 1.000 habitantes & 0 & 0 & 0 & 8 & 5 \\
\hline $\begin{array}{l}\mathrm{N}^{\circ} \text { de leitos hospitalares } \mathrm{p} / 1.000 \\
\text { hab. }\end{array}$ & 0 & 1 & 5 & 6 & 7 \\
\hline $\begin{array}{l}\mathrm{N}^{\circ} \text { de hab./estabelecimento de } \\
\text { saúde }\end{array}$ & 10 & 2 & 0 & 0 & 1 \\
\hline \multicolumn{6}{|l|}{ Escolarização } \\
\hline 0 a 6 anos & 0 & 3 & 9 & 1 & 1 \\
\hline 7 a 14 anos & 0 & 7 & 4 & 2 & 2 \\
\hline 15 a 17 anos & 1 & 7 & 5 & 0 & 0 \\
\hline 18 a 24 anos & 0 & 6 & 6 & 0 & 1 \\
\hline 25 anos ou mais & 0 & 3 & 9 & 1 & 1 \\
\hline \multicolumn{6}{|l|}{ Alfabetização } \\
\hline 10 a 14 anos & 1 & 11 & 1 & 0 & 0 \\
\hline 15 a 19 anos & 4 & 7 & 2 & 0 & 0 \\
\hline 20 anos ou mais & 1 & 10 & 2 & 0 & 0 \\
\hline Escolaridade & & 6 & 7 & 0 & 0 \\
\hline Analfabetismo funcional & 5 & 8 & 0 & 0 & 0 \\
\hline $\begin{array}{l}\text { Famílias atendidas com programas } \\
\text { sociais }\end{array}$ & 6 & & 6 & 0 & 1 \\
\hline \multicolumn{6}{|l|}{$\begin{array}{l}\text { Adequação de moradia nos } \\
\text { domicílios }\end{array}$} \\
\hline $\begin{array}{l}\text { Domicílio com banheiro e água } \\
\text { encanada }\end{array}$ & 4 & 5 & 4 & 0 & 0 \\
\hline $\begin{array}{l}\text { Domicílios urbanos c/ serviço de } \\
\text { coleta de lixo }\end{array}$ & 11 & 2 & 0 & 0 & 0 \\
\hline Domicílio com energia elétrica & 10 & 2 & 1 & 0 & 0 \\
\hline Domicílio com densidade >2 & 6 & 4 & 3 & 0 & 0 \\
\hline $\begin{array}{l}\text { Mortalidade por homicídio } \\
\text { (agressões) }\end{array}$ & 13 & 0 & 0 & 0 & 0 \\
\hline $\begin{array}{l}\text { Mortalidade por acidente de } \\
\text { transporte }\end{array}$ & 12 & 1 & 0 & 0 & 0 \\
\hline
\end{tabular}


O grupo de índices da dimensão social está relacionado à melhoria da qualidade de vida e justiça social, acesso aos serviços básicos de saúde, ao nível de educação e às condições básicas de sobrevivência humana. Assim, observou-se na Tabela 6 que o índice referente à saúde como: a imunização contra doenças infecciosas infantis; os números de médicos e os números de leitos hospitalares por habitantes apresentam uma situação de alerta para crítica, muito abaixo do ideal.

Quanto ao índice de escolarização, 30\% dos municípios da amostra também apresentam uma situação de alerta para crítica. Ademais, nota-se a falta de informação para algumas variáveis dos índices de saúde e escolarização, o que prejudica a análise desses dados, sendo necessária uma atenção especial por parte das políticas públicas para essas áreas respectivamente. Em relação aos outros índices, verifica-se que tiveram um comportamento aceitável para ideal.

Tabela 7 - Índice de Desenvolvimento Sustentável - Dimensão SOCIAL.

\begin{tabular}{|c|c|c|}
\hline MUNICÍPIOS PARAENSES & IDSM / COLORAÇÃO & CLASSIFICAÇÃO \\
\hline Altamira & 0,6434 & Aceitável \\
\hline Canãa dos Carajás & 0,7065 & Aceitável \\
\hline Dom Eliseu & 0,5980 & Aceitável \\
\hline Juruti & 0,6090 & Aceitável \\
\hline Óbidos & 0,6024 & Aceitável \\
\hline Paragominas & 0,6072 & Aceitável \\
\hline Redenção & 0,6445 & Aceitável \\
\hline Santana do Araguaia & 0,6058 & Aceitável \\
\hline Santarém & 0,6027 & Aceitável \\
\hline Tailândia & 0,5368 & Aceitável \\
\hline Tucumã & 0,6739 & Aceitável \\
\hline Tucuruí & 0,6411 & Aceitável \\
\hline Xinguara & 0,6777 & Aceitável \\
\hline
\end{tabular}

Fonte: Dados da Pesquisa (2016).

A partir da análise em conjunto dos índices de sustentabilidade social referentes aos 13 municípios paraenses pesquisados (Tabela 7), verificou-se que todos apresentaram o nível de sustentabilidade social aceitável, variando entre 0,5368 a 0,7065.

A dimensão econômica está relacionada ao desempenho econômico e financeiro e aos rendimentos da população. Na Tabela 8 são apresentados os valores encontrados para essa dimensão. 
Tabela 8 - Nível de Sustentabilidade ECONÔMICA dos municípios por variável.

\begin{tabular}{|c|c|c|c|c|c|}
\hline \multirow{2}{*}{$\begin{array}{c}\text { DIMENSÃO ECONÔMICA } \\
13 \text { municípios do estado do Pará } \\
\text { VARIÁVEIS }\end{array}$} & \multicolumn{5}{|c|}{$\begin{array}{l}\text { Nível de Sustentabilidade ECONÔMICA dos } 13 \\
\text { municípios paraenses, por variável (unidade) }\end{array}$} \\
\hline & IDEAL & ACEITÁVEL & ALERTA & CRÍTICA & $\begin{array}{c}\text { SEM } \\
\text { INFORM. }\end{array}$ \\
\hline Produto Interno Bruto per capita & 1 & 0 & 1 & 11 & 0 \\
\hline Participação da indústria no PIB & 2 & 1 & 5 & 5 & 0 \\
\hline Saldo da balança comercial & 0 & 0 & 0 & 13 & 0 \\
\hline \multicolumn{6}{|l|}{ Renda familiar per capita } \\
\hline Até 1/4 (salário mínimo) & 4 & 7 & 1 & 1 & 0 \\
\hline Mais de $1 / 4$ a $1 / 2$ (salário mínimo) & 1 & 3 & 7 & 2 & 0 \\
\hline Mais de $1 / 2$ a 1 (salário mínimo) & 0 & 2 & 8 & 3 & 0 \\
\hline Mais de 1 a 2 (salário mínimo) & 0 & 2 & 9 & 2 & 0 \\
\hline Mais de 2 a 3 (salário mínimo) & 0 & 1 & 8 & 4 & 0 \\
\hline Mais de 3 (salário mínimo) & 0 & 0 & 0 & 9 & 4 \\
\hline Renda per capita & 0 & 6 & 4 & 3 & 0 \\
\hline $\begin{array}{l}\text { Rendimentos provenientes do } \\
\text { trabalho }\end{array}$ & 10 & 3 & 0 & 0 & 0 \\
\hline $\begin{array}{l}\text { Índice de Gini (distribuição do } \\
\text { rendimento) }\end{array}$ & 0 & 6 & 7 & 0 & 0 \\
\hline
\end{tabular}

Observa-se que os municípios paraenses da pesquisa, quanto aos indicadores do PIB e saldo da balança comercial, sinalizam um estado crítico do desenvolvimento econômico local, mostrando um desequilíbrio da participação da indústria e dos demais setores na formação do PIB dos municípios pesquisados. O saldo da balança comercial está relacionado com as importações e exportações para fora do País, o que, para o grupo dos municípios pesquisados, é pouco significativo. Além disso, esses índices apresentam uma disparidade entre os setores produtivos, sendo necessário manter a diversificação das atividades produtivas, levando em consideração a rentabilidade econômica de cada localidade.

A partir da análise em conjunto dos índices de sustentabilidade econômica referentes aos 13 municípios paraenses pesquisados, verificou-se que quase todos apresentaram o nível para o estado de alerta, variando entre 0,2379 a 0,5748 .

Tabela 9 - Índice de Desenvolvimento Sustentável - Dimensão ECONÔMICA.

\begin{tabular}{|c|c|c|}
\hline MUNICÍPIOS PARAENSES & IDSM / COLORAÇÃO & CLASSIFICAÇÃO \\
\hline Altamira & 0,3459 & Alerta \\
\hline Canãa dos Carajás & 0,5748 & Aceitável \\
\hline Dom Eliseu & 0,3247 & Alerta \\
\hline Juruti & 0,3552 & Alerta \\
\hline Óbidos & 0,2379 & Crítica \\
\hline Paragominas & 0,3290 & Alerta \\
\hline Redenção & 0,3562 & Alerta \\
\hline Santana do Araguaia & 0,3626 & Alerta \\
\hline Santarém & 0,3450 & Alerta \\
\hline Tailândia & 0,3372 & Alerta \\
\hline Tucumã & 0,3536 & Alerta \\
\hline Tucuruí & 0,4186 & Alerta \\
\hline Xinguara & 0,3798 & Alerta \\
\hline
\end{tabular}


Fonte: Dados da Pesquisa (2016)

Nota-se na Tabela 9 que o município de Canãa dos Carajás apresenta o maior índice do grupo quanto à dimensão econômica, mas o seu índice de 0,5748 ainda é relativamente baixo para o desempenho aceitável. Entretanto, o município de Óbidos apresentou o menor índice da amostra, com 0,2379, a partir de uma avaliação crítica. Já para o restante da amostra, os índices ficaram mais próximos da limítrofe do alerta para o crítico, refletindo um cenário econômico estagnado.

Alguns fatores podem permitir uma reflexão para melhorar o desenvolvimento das políticas públicas para o crescimento da dimensão econômica, como o incentivo à inserção de novas empresas locais para geração de empregos e o desenvolvimento das potencialidades locais, como a agricultura, pesca e a pecuária.

Portanto, essa dimensão exige um estudo maior de alternativas justas que propiciem o equilíbrio da renda da população e estimulem a prática de importações e exportações, por meio de incentivo de instalações de indústrias nos municípios.

A dimensão ambiental está relacionada ao uso adequado dos recursos naturais, à degradação do ecossistema e à preservação e conservação do meio ambiente. Seus índices estão relacionados à qualidade, tratamento, consumo médio e formas de abastecimento de água por domicílio, tipos de tratamento sanitário por habitação e acesso à coleta de lixo urbano. Os resultados para dimensão ambiental apresentaram uma avaliação positiva, conforme descrição na Tabela 10 abaixo:

Tabela 10 - Nível de Sustentabilidade AMBIENTAL dos 13 municípios por variável.

\begin{tabular}{|c|c|c|c|c|c|}
\hline \multirow{2}{*}{$\begin{array}{c}\text { DIMENSÃO AMBIENTAL } \\
13 \text { municípios do estado do Pará } \\
\text { VARIÁVEIS }\end{array}$} & \multicolumn{5}{|c|}{$\begin{array}{l}\text { Nível de Sustentabilidade AMBIENTAL dos } 13 \\
\text { municípios paraenses, por variável (unidade) }\end{array}$} \\
\hline & IDEAL & $\begin{array}{l}\text { ACEITÁV } \\
\text { EL }\end{array}$ & ALERTA & CRÍTICA & $\begin{array}{l}\text { SEM } \\
\text { INFORM }\end{array}$ \\
\hline \multicolumn{6}{|l|}{ Qualidade das águas } \\
\hline Aferição de cloro residual & 12 & 0 & 0 & 1 & 0 \\
\hline $\begin{array}{l}{ }^{*} \text { Incidência de amostras de cloro } \\
\text { residual fora do padrão }\end{array}$ & 13 & 0 & 0 & 0 & 0 \\
\hline Aferição de turbidez & 10 & 1 & 2 & 0 & 0 \\
\hline $\begin{array}{l}{ }^{*} \text { Incidência de amostras com } \\
\text { turbidez fora do padrão }\end{array}$ & 12 & 0 & 1 & 0 & 0 \\
\hline Aferição de coliformes fecais & 12 & 0 & 1 & 0 & 0 \\
\hline $\begin{array}{l}{ }^{*} \text { Incidência de amostras com } \\
\text { coliformes fecais fora do padrão }\end{array}$ & 13 & 0 & 0 & 0 & 0 \\
\hline \multicolumn{6}{|l|}{ Tratamento das Águas } \\
\hline Tratada em ETAs & 2 & 0 & 0 & 0 & 11 \\
\hline Tratada por desinfecção & 4 & 0 & 0 & 0 & 9 \\
\hline Consumo médio per capita de água & 13 & 0 & 0 & 0 & 0 \\
\hline \multicolumn{6}{|l|}{$\begin{array}{l}\text { Acesso ao sistema de abastecimento } \\
\text { de água }\end{array}$} \\
\hline Rede geral urbana & 2 & 4 & 3 & 4 & 0 \\
\hline Poço ou nascente urbana & 5 & 1 & 1 & 5 & 1 \\
\hline Outro tipo urbano & 5 & 7 & 1 & 0 & 0 \\
\hline
\end{tabular}




\begin{tabular}{|l|c|c|c|c|c|}
\hline $\begin{array}{l}\text { Tipo de esgotamento sanitário por } \\
\text { domicílio }\end{array}$ & & & & & \\
\hline Rede geral de esgoto urbano & 0 & 1 & 1 & 11 & 0 \\
\hline Fossa séptica urbana & 0 & 1 & 2 & 10 & 0 \\
\hline Outro tipo urbano & 0 & 0 & 0 & 0 & 0 \\
\hline $\begin{array}{l}\text { Não possui esgotamento sanitário } \\
\text { urbano }\end{array}$ & 9 & 4 & 0 & 0 & 0 \\
\hline Acesso à coleta de lixo urbano e rural & & & & & \\
\hline Lixo coletado urbano & 5 & 5 & 3 & 0 & 0 \\
\hline Lixo queimado ou enterrado urbano & 11 & 2 & 0 & 0 & 0 \\
\hline Outro destino urbano & 13 & 0 & 0 & 0 & 0 \\
\hline
\end{tabular}

Fonte: Dados da Pesquisa (2016).

Observa-se, na Tabela 10 acima, a falta de informação para os indicadores de Tratamento das Águas e o estado crítico para o indicador Tipo de Esgotamento Sanitário por domicílio. Considerando que essas informações são de muita relevância para análise da sustentabilidade local, a ausência desses dados prejudicou o índice de desenvolvimento sustentável municipal para o grupo da dimensão ambiental. Outro fator de relevância é a taxa de desmatamento que o sistema IDSM não possui, deixando o resultado fragilizado. Já para as variáveis coletadas na dimensão ambiental, o desempenho apresentouse aceitável para ideal, de acordo com os dados na Tabela 11:

Tabela 11 - Índice de Desenvolvimento Sustentável - Dimensão AMBIENTAL.

\begin{tabular}{|c|c|c|}
\hline MUNICÍPIOS PARAENSES & IDSM/COLORAÇÃO & CLASSIFICAÇÃO \\
\hline Altamira & 0,6097 & Aceitável \\
\hline Canãa dos Carajás & 0,7238 & Aceitável \\
\hline Dom Eliseu & 0,7724 & Ideal \\
\hline Juruti & 0,7447 & Aceitável \\
\hline Óbidos & 0,7317 & Aceitável \\
\hline Paragominas & 0,6670 & Aceitável \\
\hline Redenção & 0,6509 & Aceitável \\
\hline Santana do Araguaia & 0,6371 & Aceitável \\
\hline Santarém & 0,7872 & Ideal \\
\hline Tailândia & 0,7283 & Aceitável \\
\hline Tucumã & 0,6835 & Aceitável \\
\hline Tucuruí & 0,7340 & Aceitável \\
\hline Xinguara & 0,6215 & Aceitável \\
\hline
\end{tabular}

Fonte: Dados da Pesquisa (2016).

Na Tabela 11, verificou-se que todos os municípios da amostra apresentaram o nível de sustentabilidade ambiental aceitável, com índices variando entre 0,6097 e 0,7447. Destacam-se dois municípios avaliados com desempenho ideal: Dom Eliseu e Santarém, cujos valores variam, respectivamente, entre 0,7724 e 0,7872 . 
As informações produzidas para o grupo da dimensão ambiental oferecem subsídios para entender aspectos importantes do processo de degradação ambiental, derivados da falta de infraestrutura, comportamentos e hábitos inadequados, formas de agir e pensar da população, no sentido de compreender e respeitar as limitações dos recursos naturais e o tempo necessário para recomposição do meio ambiente. Portanto, essas informações são relevantes para a criação de políticas e mecanismos voltados à proteção, conservação e preservação do meio ambiente que proporcionem melhor qualidade de vida e ambiental.

Na Tabela 12, apresenta-se o resultado final para os 13 municípios avaliados no ano de 2010, em distinção hierárquica.

Tabela 12 - Hierarquização do IDSM Final dos municípios paraenses.

\begin{tabular}{|c|c|c|}
\hline MUNICÍPIOS PARAENSES & COLORAÇÃO & CLASSIFICAÇÃO \\
\hline Óbidos & $\mathbf{0 , 3 7 7 3}$ & Alerta \\
\hline Tucumã & $\mathbf{0 , 4 1 2 6}$ & Alerta \\
\hline Santana do Araguaia & $\mathbf{0 , 4 2 0 4}$ & Alerta \\
\hline Tailândia & $\mathbf{0 , 4 2 1 4}$ & Alerta \\
\hline Dom Eliseu & $\mathbf{0 , 4 2 6 5}$ & Alerta \\
\hline Juruti & $\mathbf{0 , 4 2 7 6}$ & Alerta \\
\hline Redenção & $\mathbf{0 , 4 3 5 1}$ & Alerta \\
\hline Paragominas & $\mathbf{0 , 4 6 0 3}$ & Alerta \\
\hline Altamira & $\mathbf{0 , 4 6 7 6}$ & Alerta \\
\hline Xinguara & $\mathbf{0 , 4 7 0 1}$ & Alerta \\
\hline Canãa dos Carajás & $\mathbf{0 , 4 9 6 6}$ & Alerta \\
\hline Santarém & $\mathbf{0 , 5 0 1 5}$ & Aceitável \\
\hline Tucuruí & $\mathbf{0 , 5 3 1 0}$ & Aceitável \\
\hline
\end{tabular}

Fonte: Dados da Pesquisa (2016).

De acordo com o resultado final, os municípios paraenses da amostra apresentam resultados pouco satisfatórios, visto que apenas dois municípios apresentaram um índice final aceitável e todos os outros se encontram em nível de alerta. O município que apresentou o melhor índice foi Tucuruí com quantitativo de 0,5310, o que reflete uma avaliação aceitável. O segundo melhor colocado foi o de Santarém, que com um índice de 0,5015, apresenta uma classificação aceitável. Em contrapartida, o pior índice de sustentabilidade foi revelado pela cidade de Óbidos, cujo IDSM é de 0,3773.

Observa-se que os fatores que fizeram Tucuruí e Santarém ficarem mais bem classificadas no IDSM foram as pontuações nas dimensões ambiental, social e cultural, enquanto que a pontuação na dimensão político-institucional do município de Óbidos foi a mais baixa da pesquisa, resultando no pior índice.

A pesquisa demonstrou que a Certificação Verde emitida pelo PMV não tem efeito no IDSM dos municípios paraenses pesquisados, por dois fatores: primeiro, quanto à dimensão ambiental, pois não apresenta dentro de suas variáveis a taxa de redução de desmatamento do município. Segundo, a Certificação Verde iniciou em 2009, bem como os seus benefícios e, dificilmente, os benefícios dessa certificação surtiriam efeitos em 2010. Normalmente é necessário um período de tempo maior. 


\subsection{RELAÇÃO DO IDSM COM OS FINANCIAMENTOS BANCÁRIOS}

Os valores encontrados para o IDSM dos municípios da amostra, por meio da metodologia de Martins e Cândido (2008), foram relacionados com os financiamentos bancários (públicos e privados), a fim de se verificar o grau de correlação entre eles. Para os índices dos financiamentos bancários, foi calculada a variável do recurso monetário mediante a somatória dos financiamentos liberados mensalmente no ano de 2010, pelo total da população dos municípios da pesquisa, conforme apresentado na Tabela 13.

Tabela 13 - Hierarquização dos IDSM e os Financiamentos liberados pelas Instituições Financeiras Bancárias (Públicas e Privadas) dos municípios avaliados (2010).

\begin{tabular}{|c|c|c|c|c|}
\hline Municípios & IDSM/COLOR & FPB/hab.(R\$) & FPV/hab.(R\$) & Ftotal/hab.(R\$) \\
\hline Óbidos & 0,3773 & $15.003,70$ & - & $15.003,70$ \\
\hline Tucumã & 0,4126 & $19.879,97$ & $2.484,87$ & $22.364,84$ \\
\hline Santana do Araguaia & 0,4204 & $15.831,66$ & 846,61 & $16.678,27$ \\
\hline Tailândia & 0,4214 & $12.746,90$ & - & $12.746,90$ \\
\hline Dom Eliseu & 0,4265 & $5.572,04$ & $1.685,52$ & $7.257,55$ \\
\hline Juruti & 0,4276 & $9.660,51$ & & $9.660,51$ \\
\hline Redenção & 0,4351 & $33.968,58$ & $7.292,86$ & $41.261,45$ \\
\hline Paragominas & 0,4603 & $21.202,79$ & $4.126,09$ & $25.328,88$ \\
\hline Altamira & 0,4676 & $23.650,66$ & $2.140,52$ & $25.791,18$ \\
\hline Xinguara & 0,4701 & $44.269,47$ & $8.444,38$ & $52.713,84$ \\
\hline Canaã dos Carajás & 0,4966 & $8.669,39$ & - & $8.669,39$ \\
\hline Santarém & 0,5015 & $21.520,40$ & $3.651,90$ & $25.172,29$ \\
\hline Tucuruí & 0,5310 & $31.840,19$ & $2.930,66$ & $34.770,84$ \\
\hline
\end{tabular}

Fonte: Dados da Pesquisa (2016); Banco Central (2016).

A Tabela 13 revela que os dois municípios que se destacam no IDSM na avaliação aceitável são Santarém e Tucuruí, o que demonstra que os recursos liberados são significativos com relação ao grupo da amostra. Os recursos liberados para o município de Óbidos, que apresentou o menor IDSM da amostra, refletiram o seu baixo desempenho quanto ao desenvolvimento sustentável.

Outros três municípios apresentaram pouca movimentação de financiamento, como Dom Elizeu, Juruti e Canãa dos Carajás. Portanto, para melhor compreender a relação dessas duas variáveis (IDSM e Financiamentos Bancários), aplicou-se a Correlação de Pearson, conforme demonstrado na Tabela 14.

Tabela 14 - Matriz de Correlação de Pearson entre os índices de financiamento bancário (público, privado e total) com o índice de desenvolvimento da sustentabilidade para os municípios paraenses no ano de 2010.

\begin{tabular}{|c|c|c|c|}
\hline Variáveis & FPB (R\$/hab.) & FPV (R\$/hab.) & Ftotal (R\$/hab.) \\
\hline \multirow{2}{*}{ IDSM } & $\mathbf{0 , 3 7 6}$ & $\mathbf{0 . 1 5 9}$ & $\mathbf{0 . 3 6 7}$ \\
\cline { 2 - 4 } & $0.206^{\mathrm{NS}}$ & $0.682^{\mathrm{NS}}$ & $0.218^{\mathrm{NS}}$ \\
\hline
\end{tabular}

Fonte: Banco Central (2016); Atlas Brasil (2016).

HO: Existe correlação significativa entre as variáveis $(p<0.10)$.

Nota: Teste de Correlação de Pearson. (COHEN, 1988).

Verifica-se na matriz de correlação que o IDSM apresentou correlação positiva média com os índices de financiamento público $(R 2=0.376)$ e o índice de financiamento total $(R 2=0.367)$, enquanto que o índice de financiamento privado $(\mathrm{R} 2=0.159)$ apresentou correlação positiva fraca. Nota-se que para todos os financiamentos destaca-se a observação "NS", o que significa que o nível de confiança ficou agravado ( $p>0.10)$. Isto é, se a correlação é não significativa (NS) estatisticamente, não há relação entre 
o índice de financiamento e o IDSM, mas, no entanto, não descarta a possibilidade de relação para um aumento na coleta da amostra, que pode influenciar o nível de confiança dos resultados, diminuindo os erros e validando a real correlação dos financiamentos bancários.

\section{CONCLUSÕES}

Foram identificados os níveis de sustentabilidade social, demográfica, econômica, político-institucional, ambiental e cultural de municípios paraenses, constatando-se que os municípios da amostra apresentam índices de sustentabilidade entre 0,3773, considerado como nível de Alerta, e 0,5310 como nível Aceitável, segundo a metodologia utilizada na pesquisa.

Observou-se que os 13 municípios paraenses selecionados, dos quais 11 possuem a certificação de Municípios Verdes, não são sustentáveis de acordo com a metodologia de Martins e Cândido (2008). Ressalva-se, contudo, que a taxa de desmatamento no município não aparece entre as variáveis da dimensão ambiental do sistema de indicadores do IDSM, deixando essa dimensão fragilizada, haja vista a importância dessa mensuração para a questão da sustentabilidade municipal.

Apesar das dificuldades atuais apresentadas pelos municípios paraenses, o Programa Município Verde (PMV) é um incentivo real para essa mudança, pois mobiliza e fiscaliza ações sustentáveis quanto à preservação da floresta e regularização do território municipal, promovendo: a valorização dos produtos de origem legal; a liberação de maior crédito pelo Governo Federal para os municípios ambientalmente responsáveis; maior incentivo fiscal para redução de impostos (ICMS Verde) e a atração de maiores investimentos para o desenvolvimento de projetos sustentáveis.

Entre a correlação do IDSM dos municípios verdes paraenses com os financiamentos bancários, o financiamento bancário público foi o que apresentou uma possibilidade de correlação para a promoção do desenvolvimento sustentável municipal, pois apresenta maiores linhas de financiamentos para o desenvolvimento sustentável, muitas delas oriundas do BNDES. Portanto, apesar da presença de instituições financeiras bancárias nos municípios paraenses, com propostas de desenvolvimento econômico local, a correlação apresentou-se com valores poucos significativos, principalmente no caso dos bancos privados.

Tal situação aponta a necessidade de os bancos reverem suas linhas de financiamentos de forma mais específica para o desenvolvimento sustentável dos municípios paraenses, promovendo o aumento no nível de sustentabilidade municipal, no sentido de melhorar a qualidade de vida da população local com incentivo para novos empreendedores, a fim de se obter recursos financeiros para o desenvolvimento econômico, social, cultural e ambiental, objetivando melhores índices de sustentabilidade.

Para futuras pesquisas, sugere-se a investigação de todos os municípios paraenses, pois, de acordo com a metodologia de Pearson, o aumento na coleta da amostra influencia o nível de confiança dos resultados, diminuindo os erros e validando a real correlação dos financiamentos bancários para promoção e manutenção, não só do ecossistema, mas, principalmente, do desenvolvimento humano, político e econômico dessas localidades.

\section{NOTAS}

10 ambientalismo moderado se fundamenta no conceito de ecodesenvolvimento que evoluiu para o conceito de desenvolvimento sustentável. $2<$ http://municipiosverdes.com.br>.

\section{REFERÊNCIAS}

BANCO MUNDIAL. Relatório sobre o Desenvolvimento Mundial 2009: a geografia econômica em transformação - visão geral. Washington, DC, 2008.

BARBIERI, C. J. Desenvolvimento e meio ambiente. Petrópolis: Vozes; 2001. 
BRAGA, C. O. da S. Protocolo verde: as instituições financeiras e a promoção da sustentabilidade ambiental no Brasil. UFPE, 2014. Disponível em: <http://repositorio.ufpe.br/handle/123456789/11722>. Acesso em: 12 ago. 2015.

BRASIL. Presidência da República. Lei Complementar no 124, de 3 de janeiro de 2007. Institui a Sudam e dispõe sobre o Fundo de Desenvolvimento da Amazônia - FDA. Disponível em: <http://www.planalto.gov.br/ccivil_03/ leis/LCP/Lcp124.htm>. Acesso em: 14 fev. 2017.

COHEN, J. Statistical Power Analysis for the Behavioral Sciences. Hillsdale: Erlbaum, 1988.

DE FREITAS, L. P. Um Ensaio sobre o Projeto do Fundo Amazônia: Secretaria de Estado do Meio Ambiente do Pará (SEMA-Pará). Fundo Amazônia: evolução recente e perspectivas, p. 216, 2013.

ELY, A. Desenvolvimento sustentado: uma abordagem holística e integrada da política, da economia, da natureza e da sociedade. Porto Alegre, FEPLAM, 1992.

FIALHO, F. A. P. et al. Gestão da sustentabilidade na era do conhecimento. Florianópolis: Visual books, 2008.

GONÇALVES, C. Avaliação de Projetos - Fundo Amazônia Projeto “Sementes do Portal”. Disponível em: <http:// www.usp.br/mudarfuturo/cms/wp-content/uploads/Fundo-Amaz\%C3\%B4nia-Projeto-Sementes-do-Portal-VII. pdf>. Acesso em: 15 ago. 2016.

MARTINS, M. F.; CÂNDIDO, G. A. Índice de Desenvolvimento Sustentável para Municípios (IDSM): metodologia para análise e cálculo do IDSM e classificação dos níveis de sustentabilidade - uma aplicação no estado da Paraíba. João Pessoa: SEBRAE, 2008.

OLIVEIRA, R. C. de. et al. Desmatamento e crescimento econômico no Brasil: uma análise da curva de Kuznets ambiental para a Amazônia legal. Revista de economia e sociologia rural, v. 49, n. 3, p. 709-739, 2011. Disponível em: <http://www.scielo.br/scielo.php?script=sci_serial\&pid=0103-2003\&lng=en\&nrm=iso>. Acesso em: 14 mar. 2017.

PMV. Programa Municípios Verdes. Disponível em: <http://www.investpara.pa.gov.br/pt-br/node/68>. Acesso em: 16 jun. 2016.

ROHDE, G. M. Mudanças de paradigma e desenvolvimento sustentado. Desenvolvimento e natureza: estudos para uma sociedade sustentável, v. 2, p. 41-53, 1999.

SANTOS, J. E. dos. Mercado financeiro brasileiro. São Paulo: Atlas, 1999.

VALE, V. P. C. Desenvolvimento Sustentável e as suas relações com os financiamentos bancários: um estudo aplicado aos municípios verdes do estado do Pará. Dissertação (Mestrado), UNAMA, Belém, 2017.

VAN BELLEN, H. M. Indicadores de sustentabilidade: uma análise comparativa. FGV, Editora, 2005.

WAQUIL, P. D. et al. Avaliação de desenvolvimento territorial em quatro territórios rurais no Brasil. Porto Alegre: PGDR/UFRGS, 2006.

WHATELY, M.; CAMPANILI, M. Programa Municípios Verdes: lições aprendidas e desafios para 2013/2014. Belém: Governo do Estado, Programa Municípios Verdes, 2013. 